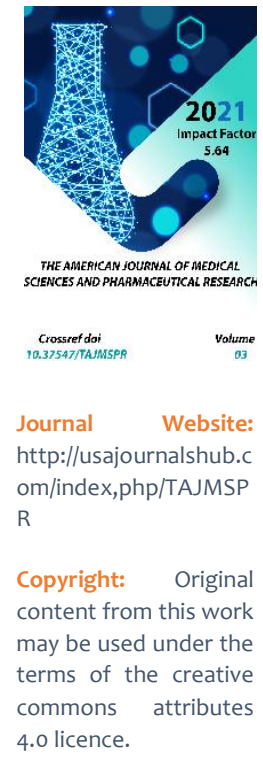

\title{
Features Of Anemia In Patients With Chronic Liver Diseases
}

Ismatova Mehriniso Nasritdinovna

Bukhara State Medical Institute, Uzbekistan

Mukhammedzhanova Mastura Hayatovna

Bukhara State Medical Institute, Uzbekistan

\section{ABSTRACT}

The features of the epidemiology, clinical picture, diagnosis and treatment of chronic diffuse liver diseases in combination with anemia are considered according to the literature on this problem.

It has been shown that anemia aggravates the course, causes progression and worsens the prognosis of chronic diffuse liver diseases. The incidence of anemia in chronic diffuse liver diseases reaches $80 \%$. Disclosure of the mechanisms of anemia in chronic diffuse liver diseases is necessary for timely correction and prevention of the progression of both pathological changes in red blood and underlying liver disease.

\section{KEYWORDS}

Chronic hepatitis, liver cirrhosis, anemia.

\section{INTRODUCTION}

In recent decades, in many countries of the world, there has been a tendency towards an increase in the number of patients with chronic diffuse liver diseases (CDLD), especially among 
young people of working age. CDDD are characterized by a severe course, poor prognosis and are one of the main causes of disability in patients in developed countries [2]. According to the $\mathrm{WHO}$, cirrhosis of the liver (LC) is the eighth leading cause of death. Therefore, the study of the features of epidemiology, clinic, diagnosis, treatment of these diseases is one of the urgent problems of modern hepatology.

Much attention is paid to alcoholic and alcoholic-viral liver diseases, which are characterized by the predominance of chronic forms, rapid progression with an outcome in LC and hepatocellular carcinoma. Alcoholic liver disease acquires special social significance in Russia, which is in the leading group of states in the consumption of alcoholic beverages [7, 10].

In the last decade, viral hepatitis with bloodborne transmission has acquired particular importance in Russia due to the spread of drug addiction. Up to $80 \%$ of injecting drug users are infected with viruses $B, C, D$ or several at the same time. The problem of mixed hepatitis $B+$ $C, B+D$, characterized by a variety of clinical manifestations, difficulties in early diagnosis, therapy, and earlier formation of cirrhosis, is urgent [1]. Over the years of active study, many fundamental issues of etiology, pathogenesis, diagnosis verification, course and outcomes of CDD have been disclosed. It has been proven that CDDD is a systemic pathology with frequent development of a wide range of extrahepatic manifestations, which sometimes can take a leading role in the clinical picture and cause low therapy efficiency [4]. Extrahepatic manifestations often not only mask liver damage, but also determine the prognosis of the disease [6].

The manifestation of Wilson-Konovalov disease with extrahepatic manifestations is observed in $40.8 \%$ of patients, including those with hemolytic crises in $12.7 \%$ [8].

D.T. Abdurakhmanov (2003) notes that in chronic hepatitis (CG) HBV-etiology, systemic manifestations occur in $22 \%$ of patients [3]. Extrahepatic manifestations are diagnosed in $38-48 \%$ of patients with autoimmune hepatitis and can affect almost all organs and systems [9].

Among the pathophysiological processes that create conditions for the development of extrahepatic manifestations in chronic viral hepatitis $(\mathrm{CVH})$, hypoxia plays an important role. T.V. Antonova argues that in the midst of HBV infection, at the peak of metabolic disorders, a combination of signs of hypoxic, hemic, circulatory and histotoxic (tissue) hypoxia is revealed, which, in turn, leads to anemia [7]. It is known that against the background of the progression of CDZD, there are significant shifts in red blood counts [8]. The number of erythrocytes, the level of hemoglobin, the value of the color index, the content of reticulocytes in patients with CDDD is significantly lower than in those without liver disease, and the red blood counts decrease with an increase in the activity of inflammation in the liver and the transformation of hCG into $C P$. The increase in anemia during the progression of chronic hepatitis in the circulatory system reflects the depletion of the compensatory mechanisms of the red sprout and indicates an increase in hypoxia as the pathological process in the liver worsens [10]. Hematological masks of CDZD, especially HCG and LC of viral etiology, can act for a long time as the leading clinical manifestation of the disease, without clinical and laboratory signs of liver damage.

Progressive liver damage in CDD patients and the development of fibrosis are inextricably linked with immune mechanisms [3]. The latter can determine not only the severity of 
inflammatory reactions and fibrosis, but also initiate the development of extrahepatic complications, in particular anemia. One of the mechanisms for the development of anemia in CDDD is an increase in the production of proinflammatory cytokines that can directly or indirectly alter iron metabolism, proliferation of erythroid precursors, erythropoietin production, and reduce the life span of erythrocytes [4]. O.Yu. Chernykh et al. (2006) found that anemia in patients with LC is associated with a shift in the balance of cytokines towards proinflammatory factors, and the severity of local inflammation in the liver is associated with anemia [6].

Proinflammatory cytokines are capable of inducing apoptosis, suppressing the expression of erythropoietin receptors on progenitor cells, inhibiting the formation and biological activity of erythropoietin, and leading to a decrease in the proliferation and differentiation of erythroid progenitors in CDDD. In addition, proinflammatory cytokines stimulate the production of labile free radicals (nitric oxide or superoxide anion), exerting a direct toxic effect on erythron $[8,43]$.

Anemia in patients with CDDD is traditionally referred to the group of chronic diseases anemias [3, 8]. It is characterized by a moderate decrease in hemoglobin levels and correlates with the severity of liver disease. The usually observed level of hemoglobin is in the range of $80-100 \mathrm{~g} / \mathrm{I}[4,5]$. Anemia is more often normo-, less often hypochromic. Serum iron levels and total serum iron-binding capacity are normal or slightly reduced; serum ferritin is increased, and the erythrocyte life time is shortened. A hallmark of anemia of chronic diseases, including CDDD, is a violation of iron metabolism with increased consumption of iron by the cells of the reticuloendothelial system, which causes disturbances in the circulation of iron in the body, insufficient supply of iron to erythroid progenitor cells and, consequently, ineffective erythropoiesis $[6,10]$.

Of great importance in the progression of anemia is the synthesis of erythrocytes with reduced resistance, altered lipid, protein composition. Pronounced shifts in the structure of erythrocytes against the background of CDPD lead to a change in their electrical characteristics [1, 2]. Obviously, these processes increase the aggregation and destruction of erythrocytes, which, in turn, leads to the progression of liver pathology, that is, a vicious circle is created. It can be assumed that changes in the structure of the membranes of erythrocytes, their compaction disrupt the saturation of erythrocytes with oxygen, dissociation and transfer of oxygen to other cells [5].

Some authors consider anemia of chronic diseases to be a manifestation of inadequate adaptation to the disease [5]. The direct myelotoxic effect of hepatotropic viruses and the immunological disorders caused by them are also considered as pathogenetic mechanisms of anemia in viral liver diseases. In addition, hematological manifestations of viral liver diseases are associated with direct infection with viruses of the hematopoietic organs, with replication and subsequent integration of viruses into the cells of the bone marrow, lymph nodes, spleen, and blood mononuclear cells [8].

Anemia is one of the main undesirable effects of antiviral therapy for viral CDDD, which worsen the quality of life of patients. Literature data on the incidence of cytopenic complications of specific therapy with interferon- $\alpha$ and ribavirin $\mathrm{CVH}$ demonstrate a decrease in hemoglobin content by an average of $26 \%$ from the initial level in patients receiving antiviral drugs. A decrease in the hemoglobin level of less than $100 \mathrm{~g} \mathrm{/} \mathrm{I} \mathrm{against} \mathrm{the}$ background of combined antiviral therapy is 
recorded in $7-32 \%$ of patients. Anemia associated with antiviral therapy is mainly ribavirin-induced. Most often, when taking ribavirin, erythrocyte hemolysis develops, which in $10 \%$ of patients requires a dose reduction or premature discontinuation of the drug [9]. Hemolysis is completely reversible after discontinuation of ribavirin therapy. Another cause of anemia in antiviral treatment is the ability of ribavirin metabolites to accumulate in red blood cells, reducing their lifespan. To a lesser extent, anemia is associated with the myelosuppressive effect of IFN- $\alpha$ [7].

In works of earlier years, it is described that with CDKD, normocytic anemia is detected in $30.3 \%$ of patients, microcytic anemia - in $14.4 \%$, macrocytic - in 32.6\%, absence of anemia - in $22.7 \%[6]$.

With the development of portal hypertension with portal gastropathy, there is a high risk of acute or chronic bleeding, which always lead to the development of post-hemorrhagic iron deficiency anemia (IDA) of varying degrees [3]. A separate cause of anemia is bleeding from varicose veins (varicose veins) of the esophagus and stomach, which occurs in $80 \%$ of patients with portal hypertension [10]. Mortality in bleeding from esophageal and gastric varices reaches $40 \%$, causing $50 \%$ mortality in LC [10]. The main sources of bleeding in CDZD are esophageal and gastric varices, bleeding from which makes up 20-30\% of all varicose bleeding, rupture of the esophageal mucosa in Mallory-Weiss syndrome. The third most common source of bleeding in CDDD is gastropathy. In patients with LC, the incidence of portal hypertensive gastropathy, according to the results of studies by various authors, is $45-90 \%$. Bleeding in gastropathy is often accompanied by the development of chronic IDA [10].
Folic acid deficiency, the most common cause of megaloblastic anemia in CDDD, occurs in nearly $40 \%$ of alcohol abusers. Moderate macrocytosis is usually observed in alcoholic liver disease (MCV not higher than 100-110 fL) [2]. Lack of folic acid is mainly due to the nature of the diet of patients in this group [9]. In addition, alcohol decreases methylenetetrahydrofolate reductase levels, thereby creating a functional folate deficiency. At the heart of megaloblastic changes in CDDD, to a lesser extent, there is a violation of the metabolism of vitamin B12, due to a decrease in its deposition in the liver. In addition, high-dose ethanol consumption causes direct damage to the bone marrow with the development of megaloblastic changes [5]. Refusal to drink alcohol leads to the disappearance of macrocytosis in 1 - 4 months. The presence of non-megaloblastic macrocytosis in patients with CDZD may be due to a transient increase in the number of reticulocytes in the peripheral blood as a result of a compensatory response of the bone marrow to anemia caused by blood loss from the esophageal esophageal and gastric varices, or anemia caused by hemolysis [5]. The cause of macrocytosis in CDDD is also the appearance of erythrocytes with an altered lipid composition of their membranes, which is most often observed in alcoholic liver disease. Moreover, macrocytosis in such cases persists throughout the life cycle of erythrocytes and after the complete cessation of alcohol intake.

The appearance of hemolytic anemia in CDKD may be a consequence of the instability of erythrocyte membranes, the action of serum anti-erythrocyte antibodies, or the direct effects of hepatotropic viruses on erythrocytes [1]. One of the reasons for the increased destruction of red blood cells in the spleen is congestive splenomegaly.

Hemolysis of altered erythrocytes is one of the causes of anemia in CDDD [5]. Excessive hemolysis is usually accompanied by an 
increase in the number of reticulocytes, which reflects the regenerative properties of the bone marrow. The decrease in the level of reticulocytes at the stage of CP decompensation is probably associated with a decrease in the compensatory capabilities of erythropoiesis during the progression of liver fibrosis.

Thus, many researchers most often record anemia in patients with $\mathrm{CVH}$ and $\mathrm{LC}$, regardless of etiology. The most pronounced changes in the hemogram are recorded at high clinical and biochemical activity of CG and at the stage of $\mathrm{CP}$ decompensation. Episodes of massive or occult bleeding, alcohol abuse, vitamin B12 and folate deficiencies in the presence of CDDD are factors that initiate or aggravate anemia.

Currently, the problem of anemia is extremely relevant in the management of patients with $C D D D$, since changes in red blood are quite common in the clinical picture of liver pathology. In most cases, in patients with CDZD, it is difficult to identify the leading cause of pathological changes in the red blood. Often, anemia in chronic hepatitis and, especially, LC is polyvalent, causing a wide range of qualitative and quantitative disorders in the hemogram. Changes in erythrocytes in CDZD can occur both due to the pathology of erythron, and under the influence of various factors directly on the erythrocytes of peripheral blood.

Disclosure of the mechanisms of anemia in CDZD is necessary for the timely correction and prevention of the progression of both pathological changes in red blood and the underlying liver disease.

\section{REFERENCES}

1. Antonova T.V., Baranovskaya V. B. Cardiovascular aspects of HBV infection //
Zh. infectology. - 2009. - T. 1. - No. 2/3. - S. 13 $-24$.

2. Belchenko D.I., Krivosheina E.L. Features of the bone marrow response to acute and chronic blood loss // Uspekhi sovr. natural. - 2007. - No. 9. - S. 18 - 22.

3. Bueverov A.O. Prevention and correction of hematological side effects of antiviral therapy for chronic hepatitis C // Ros. zhurn. gastroenterol., hepatol, coloproctol. - 2009. - T. 19. - No. 3. - S. 76 81.

4. Zharov S.N., Luchshev V.I., Sanin B.I. Viral hepatitis C. Actual problems of treatment // Ros. honey. zhurn. - 2010. - No. 5. - S. 22 - 25.

5. Ivashkin V.T., German E.N., Mayevskaya M.V. Latent infection with hepatitis B virus // Ros. zhurn. gastroenterol., hepatol, coloproctol. - 2008. - No. 2. - P. 4 - 11.

6. Kalinin E.Yu., Shcherbakov P.Yu., Raspereza D.V. and others. Long-term results of the use of endoscopic methods of hemostasis in gastroesophageal bleeding of portal genesis // Vestn. St. Petersburg. un-that. - 2008. - Ser. 11, no. 1. P. 72 - 76.

7. Kulikov V.E., Fishman B.B., Volkov A.V. and other Features of cerebral hemodynamics in patients with chronic diffuse liver diseases // Rats. pharmacoter. to card. 2007. - No. 4. - S. 55 - 57.

8. Kurilovich S.A., Kruchinina M.V., Generalov V.M. et al. Electrical parameters and structure of erythrocyte membranes in diffuse liver diseases // Ros. zhurn. gastroenterol., hepatol, coloproctol. 2009. - T.19. - No. 2. - S. 30 - 36.

9. Levitan B.N., Umerova A.R., Dedov A.V., Levitan G.B. Antibodies to microbial lipopolysaccharides of the intestinal ecosystem in chronic hepatitis and CP // Vestn. St. Petersburg. state honey. academy named after I. I. Mechnikov. 2008. - No. 3. - P. 135 - 138.17 
The American Journal of Medical Sciences and Pharmaceutical Research

(ISSN - 2689-1026)

Published: April 30, 2021 | Pages: 189-194

Doi: https://doi.org/10.37547/TAJMSPR/Volume03Issue04-27

IMPACT FACTOR

2021: 5.64

OCLC - 1121105510

10. Moiseev S.V. Treatment of chronic hepatitis C: results of randomized controlled trials // Inf. disease. - 2010. - T. 8.

- No. 3. - P. $52-57$ 